\title{
Brain Systems Mediating Aversive Conditioning: an Event-Related fMRI Study
}

\author{
Christian Büchel,*㧊 ond Morris,* \\ Raymond J . Dolan,*广 and Karl J . Friston* \\ *The Wellcome Department of Cognitive Neurology \\ Institute of Neurology \\ 12 Queen Square \\ London WCIN 3BG \\ tThe Royal Free Hospital School of Medicine \\ Rowland Street \\ London NW3 2PF \\ United Kingdom
}

\section{Summary}

We have used event-related functional magnetic resonance imaging (fMRI) to characterize neural responses associated with emotional learning. Employing a classical conditioning paradigm in which faces were conditioned by pairing with an aversive tone (US), we compared responses evoked by conditioned $(C S+)$ and nonconditioned (CS - ) stimuli. Pairing $50 \%$ of the CS + with the US enabled us to constrain our analysis to responses evoked by a CS + not followed by a US. Differential evoked responses, related to conditioning, were found in the anterior cingulate and the anterior insula, regions with known involvement in emotional processing. Differential responses of the amygdalae were best characterized by a time by stimulus interaction indicating a rapid adaptation of $\mathbf{C S}+-$-specific responses in this region.

\section{Introduction}

In classical conditioning paradigms, a previously neutral stimulus (conditioned stimulus or CS) comes to elicit a behavioral response through temporal pairing with an unconditioned stimulus (US). In many paradigms, the US is aversive and the behavioral response is measured in terms of changes in skin conductance (skin conductance response [SCR] or galvanic skin response [GSR]), pupil diameter, or some other measure such as freezing behavior (LeDoux, 1996). Hence, classical conditioning is a form of associative learning involving linkage between a neutral stimulus and a stimulus with high intrinsic behavioral significance.

Classical conditioning embodies elements of memory and emotional processing, a fact reflected by the associated functional anatomy. Lesion studies suggest a critical role for medial temporal lobe structures, especially the amygdala, in the acquisition of conditioned emotional responses (LaB ar and LeDoux, 1996). For example, lesions restricted to bilateral amygdalae impair the acquisition of conditioned autonomic responses but leave declarative knowledge of stimulus contingencies intact. The opposite dissociation has been reported in association with bilateral hippocampal damage (Bechara et al., 1995).

\footnotetext{
‡To whom correspondence should be addressed
}

In classical conditioning, the close temporal proximity of US and CS pairings is essential. To achieve selective conditioning of some stimuli but not others, the intertrial interval (ITI) between neutral and conditioned stimuli has to be long compared to the time between CS and US. Several functional neuroimaging studies have investigated human classical conditioning (Fredrikson et al., 1995; Schreurs et al., 1997). Positron emission tomography (PET) functional neuroimaging (Morris et al., 1997) has implicated an extended system including the pulvinar, medial thalamic nuclei, and amygdala in aversive classical conditioning of emotionally expressive faces. Notably, functional imaging studies, using PET and functional magnetic resonance imaging (fMRI), employ blocked designs, in which subjects undergo an initial conditioning block by presenting the CS with the US and the effect is then assessed in a (test) second block by presenting the CS alone (Morris et al., 1997). Unfortunately, this procedure is confounded by the fact that during the test block, when the CS is always presented alone, there may also be an extinction component where the associated functional neuroanatomy may differ from that of conditioning per se (Rolls et al., 1994).

The optimal prerequisites for studying the neurobiology of classical conditioning in humans, using functional neuroimaging, are met by event-related (mixed/single trial) fMRI technique (Buckner et al., 1996; Dale and Buckner, 1997; J osephs et al., 1997). This novel technique resembles that used to record event-related potentials in electrophysiology, where different stimuli are presented repeatedly over time. Recent methodological advances (J osephs etal., 1997) have enabled us to study evoked hemodynamic responses for the whole brain to conditioned and neutral stimuli in a manner compatible with mixed trial classical conditioning paradigms. In this study, we used a partial reinforcement strategy in which one half of the CS+ presentations were paired with the US $(C S+$ paired $)$ and the other half were not paired $\left(C S+{ }_{\text {unpaired }}\right)$, to compare evoked hemodynamic responses elicited by the CS + in the absence of the US.

\section{Results}

We chose four neutral faces, two male and two female, taken from the Ekman series (Ekman, 1982). Faces were presented for $3 \mathrm{~s}$. Subjects were scanned during two distinct phases: in an initial familiarization phase, all four faces were presented in randomized order (52 faces were presented over $10 \mathrm{~min}$ ), and in a second conditioning phase, two (one male, one female) of the four faces were paired with an unpleasant tone $(1 \mathrm{kHz})$ and consequently became CS+. The amplitude of the tone was adjusted to $10 \%$ above each subject's aversive threshold ( $\sim 100 \mathrm{~dB}$; estimated by self report during gradient switching). The $500 \mathrm{~ms}$ tone followed at the end of the 3 s presentation time of the face (Figure 1). Faces presented during the first and second phases of the experiment were identical. 
$\mathrm{CS}+{ }_{\text {paired }} \quad \mathrm{CS}+{ }_{\text {unpaired }} \quad \mathrm{CS}-\quad \mathrm{CS}+{ }_{\text {unpaired }}^{\mathrm{CS}}-\mathrm{CS}+_{\text {paired }}$
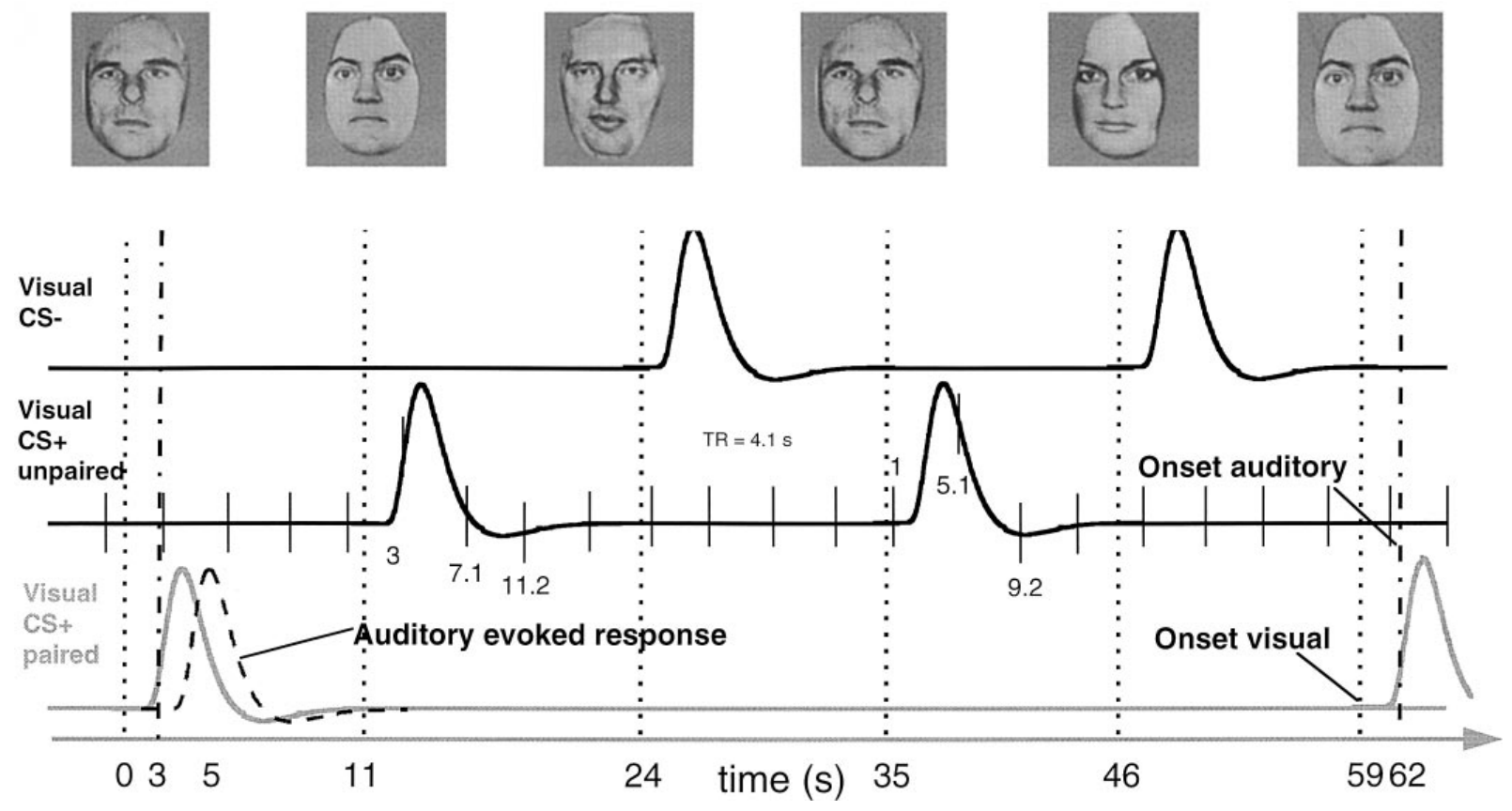

Figure 1. Illustration of the Experimental Design

The top row shows the four images used in the experiment. Two faces (first and second) were conditioned with an aversive tone. According to a $50 \%$ partial reinforcement, only one-half of the presentations were followed by the tone (e.g., first and last [CS ${ }_{\text {paired }}$ ] versus second and fourth $\left[\mathrm{CS}+_{\text {unpaired }}\right]$ presentation). Dotted and dash-dotted vertical lines indicate the onset of the visual and auditory stimuli, respectively. The three plots below the images show the modeled hemodynamic responses. Responses are time-locked to the onset of the face stimuli (CS-, CS + unpaired, and CS $\left.+_{\text {paired }}\right)$. The dashed response in the last row is an example for a hypothetical auditory evoked response. Note the overlap in time of hemodynamic responses for the paired CS + and the auditory response. The timescale indicates that the ITI was randomized to introduce a phase shift between sampling and stimulus onset (see Experimental Procedures for details). Thin vertical lines in the second row demonstrate the relation between the acquisition of $\mathrm{fMRI}$ volumes and the presentation of stimuli.

\section{Skin Conductance Responses}

The SCR time series confirmed that subjects acquired conditioned autonomic responses to the conditioned stimuli. Figure 2 (top) shows an example of the time course of the skin conductance signal for two subjects during MR scanning over a 100 s period. Statistical analysis was performed separately for data generated during scanning and for data of the remaining four subjects acquired in a separate study session. We found a significant difference between SCR for CS - compared to CS + trials for both analyses (Figure 2, bottom). This demonstrates that subjects were successfully conditioned during the fMRI experiment. As with our fMRI analysis, we analyzed only CS + that were not paired with the US.

We employed a $50 \%$ partial reinforcement strategy to assess the evoked hemodynamic response to the CS+ in the absence of the US (tone); that is, only half of the presentations of the two CS + were paired with the tone $(C S+$ paired $)$. In total, we presented 104 stimuli over $\sim 20$ min. 52 were neutral stimuli, 26 were $C S+$ paired with noise $\left(C S+{ }_{\text {paired }}\right)$ and 26 were $C S+$ not paired $\left(C S+{ }_{\text {unpaired }}\right)$ with noise. Figure 1 (top) gives an example of the scanning procedure. Using two CS + and two CS - allowed a more balanced presentation of $\mathrm{CS}-, \mathrm{CS}+_{\text {paired, }}$ and $\mathrm{CS}+{ }_{\text {unpaired }}$ in the context of the $50 \%$ partial reinforcement employed.
In contrast to evoked responses in electrophysiology, the sampling rate (i.e., TR) in event-related $f M R I$ is restricted. To characterize hemodynamic responses following an indexed stimulus, it is necessary to sample data points after the onset of many stimuli at different peri-stimulus time points. This can be achieved by the introduction of a fixed or random jitter between ITI and TR. In this experiment, we chose a random jitter, to ensure an equal distribution of data points after each event type. The ITI was randomized in the range of $3 \pm$ $0.5 \mathrm{TR}$, leading to ITIs that ranged between 10.25 and $14.35 \mathrm{~s}$. The example in Figure 1 (second row) illustrates the relationship between $\mathrm{fMRI}$ volume acquisition and trials: image volumes were acquired continuously. For example, scans took place $3,7.1$, and $11.2 \mathrm{~s}$ after the first $\mathrm{CS}+$ unpaired stimulus onset. After the second $\mathrm{CS}+$ unpaired trial, volumes were acquired 1,5.1, and $9.2 \mathrm{~s}$ after the onset of the stimulus.

\section{Functional Neuroimaging Visual and Auditory Evoked Hemodynamic Responses}

A first analysis addressed that event-related $\mathrm{FMRI}$, with a TR of $4.1 \mathrm{~s}$, is capable of detecting evoked responses elicited by sensory stimulation (i.e., visual and auditory). 

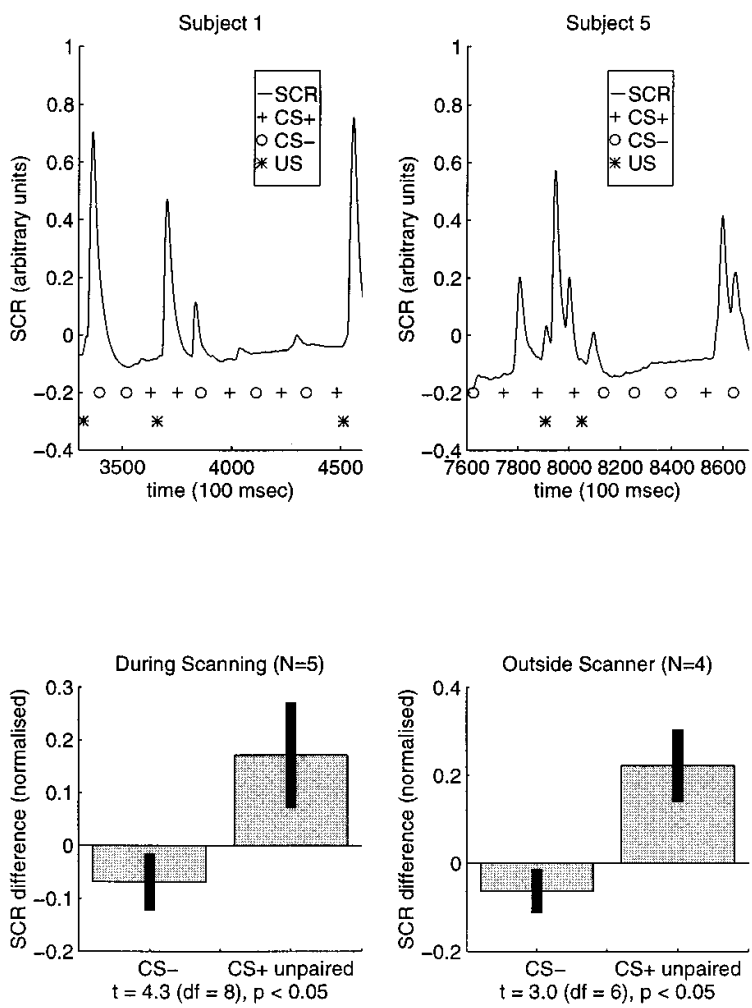

Figure 2. Skin Conductance Responses Are Shown for Two Subjects

The top graphs show the processed SCR (see text) for the presentation of face stimuli used in the experiment. Plus signs indicate the onset of a CS + stimulus. Circles indicate the onset of a CS - stimulus. Asterisks indicate the onset of the aversive tone (US), following $50 \%$ of CS + . The two bottom graphs show the statistical analysis of the SCR. The left graph demonstrates the difference and the significance of that difference for SCR data acquired during scanning. Normalized differences between a baseline and the SCR response for CS + unpaired $_{\text {and }}$ CS - are plotted with their standard errors of the mean (SEM). The right graph shows the same analysis for $S C R$ data recorded with the identical paradigm but outside the MR scanner. The difference between CS - and CS+ $+_{\text {unpaired }}$ is similar for both groups, indicating that subjects were reliably conditioned during the acquisition of $\mathrm{FMRI}$ volumes.

We compared hemodynamic responses evoked by visual face stimuli pooled over all categories $(C S+$ paired, $\mathrm{CS}+_{\text {unpaired }}$ and $\left.\mathrm{CS}-\right)$ to baseline. Activated areas were confined to bilateral striate (axial slice in Figure $3 \mathrm{~A}$ ) and extrastriate cortices. Visual evoked responses in right primary visual cortex are plotted for two of the six subjects (Figure 3A).

The second part of the analysis compared auditory evoked responses to baseline. This comparison, comprising only 26 auditory stimuli per subject, showed significant ( $p<0.05$, corrected) activations of primary auditory cortex and subcortical auditory structures. Figure 3B shows the activation of primary auditory cortex and the associated evoked hemodynamic responses in left primary auditory cortex for two subjects.

Comparison of $\mathbf{C S}+_{\text {unpaired }}$ to $\mathbf{C S}-$ Stimuli during Conditioning

In a CS $+_{\text {paired }}$ trial, the peak of the visual evoked response occurred after delivery of the auditory stimulus (first event in the third row in Figure 1). This can give rise to interaction effects in regions that respond to both the US and the CS +. To fully disambiguate the effects of $\mathrm{CS}+$ and US, we compared only responses evoked by the conditioned face stimuli when they were notfollowed by a tone $\left(\mathrm{CS}+{ }_{\text {unpaired }}\right)$. Consequently, the critical comparison in this experiment was that between the $C S+{ }_{\text {unpaired }}$ visually evoked responses and those evoked by the CS during conditioning. This comparison revealed differential activation of bilateral anterior cingulate gyri, bilateral anterior insulae, and medial parietal cortex. Further differential responses were detected in the supplementary motor area (SMA) and bilateral premotor cortices. Activation in the right $(Z=4.6)$ and left red nucleus $(Z=$ 3.6) did not reach a significance criterion of $p<0.05$ corrected, though it nevertheless achieved an uncorrected significance level at $p<0.001$. We report these results for descriptive purposes in view of the bilaterality of the activations and their previously reported involvement in conditioning (Desmond and Moore, 1991; Thompson and Krupa, 1994). Coordinates and significance levels of activations are summarized in Table 1. Figure 4A shows the location of the left anterior cingulate activation overlaid on the template T1 MRI used for spatial normalization. This coronal slice also shows activation in bilateral insulae. In Figure 4, the peri-stimulus responses are plotted for three of the nine subjects. To best characterize the data, we plotted the responses evoked by the CS - (blue \pm standard error), the response evoked by the $\mathrm{CS}+{ }_{\text {unpaired }}$ (red \pm standard error), and the difference between the two (green). Figure 4B shows activation of bilateral insulae together with the right red nucleus and individual plots of the associated peri-stimulus responses for the left anterior insula.

Rapid habituation of amygdala responses in the context of classical conditioning has been demonstrated in animal studies (Quirk et al., 1997) and human imaging (Breiter et al., 1996; Whalen et al., 1998). Such temporal effects would be disguised in a simple categorical comparison of the $\mathrm{CS}+$ unpaired and $\mathrm{CS}-$. We therefore explicitly tested for the presence of a time by event type interaction in an additional analysis. This analysis tests for areas in which neural responses evoked by $\mathrm{CS}+{ }_{\text {unpaired }}$ decrease over time, and at the same time this pattern is significantly different from the pattern for the responses evoked by the CS -. In effect, this analysis shows voxels with a differential adaptation for the $\mathrm{CS}+{ }_{\text {unpaired }}$ relative to the CS-.

In this group analysis, amygdala activations were significant bilaterally at $p<0.05$ (left $Z=4.6$ and right $Z=$ $3.2, p$ values corrected for the volume of interest and smoothness of the underlying statistical parametric map [SPM; Worsley et al., 1996]). The exact coordinates and statistics of amygdalae activations are given in Table 1.

Figure 5 shows significant voxels for this group analysis of the left amygdala on a coronal section of the T1 MRI template that was used for spatial normalization. The right amygdala response is more posterior and does not show on this selected slice. The top panels show the fitted response for the $\mathrm{CS}+{ }_{\text {unpaired }}$ trials. These data for two subjects indicate that amygdala responses to $\mathrm{CS}+$ unpaired show a rapid habituation. The associated statistics indicate the significance of the difference of this interaction in comparison to the CS - responses. 


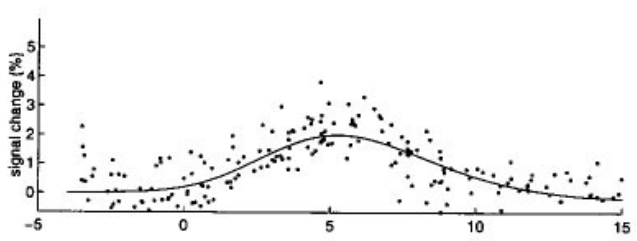

A
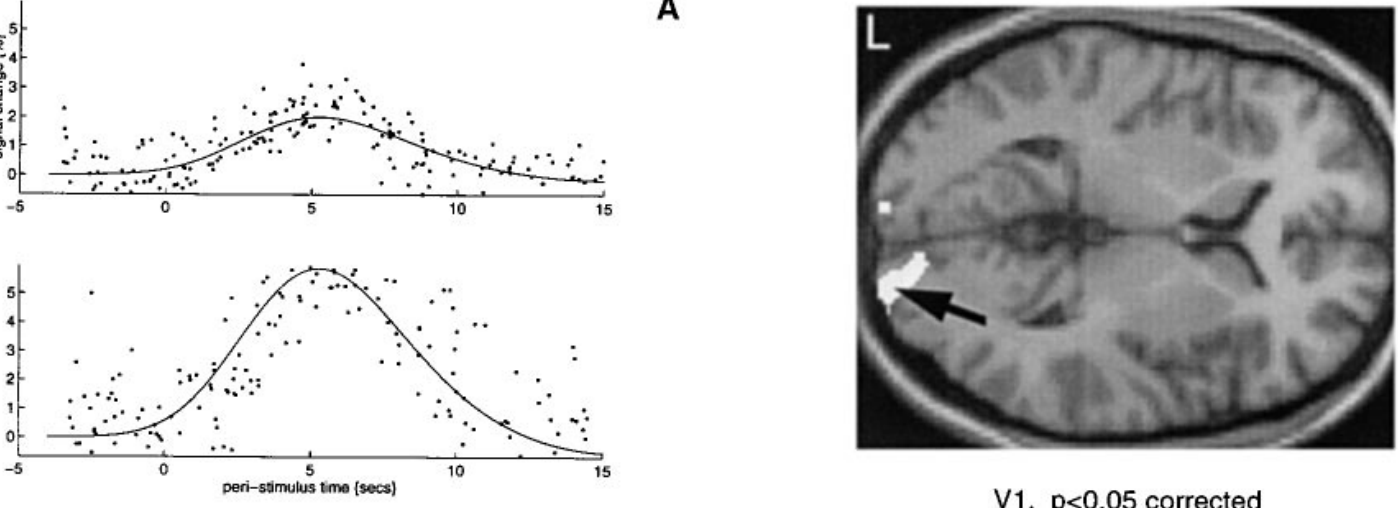

$\mathrm{V} 1, \mathrm{p}<0.05$ corrected

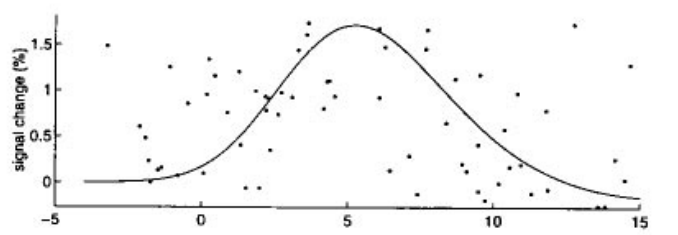

B
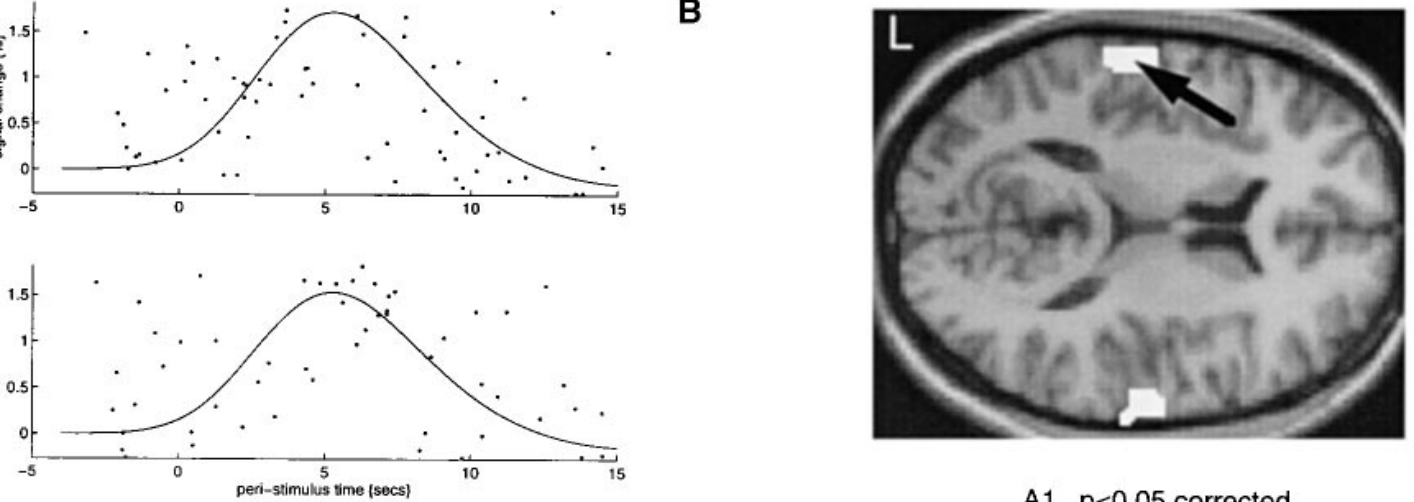

A1, $\mathrm{p}<0.05$ corrected

Figure 3. Visual and Auditory Event-Related Hemodynamic Responses

(A) Visual evoked hemodynamic responses following the presentation of faces for a subgroup of six subjects. Activations in right primary visual cortex are shown overlaid on an axial slice of the template MRI used for spatial normalization. On the left, the fitted response and adjusted data are plotted as a function of peri-stimulus time for the most significant voxel in right primary visual cortex for two subjects. Activations are thresholded at $p<0.05$ (corrected).

(B) Auditory evoked hemodynamic responses due to the presentation of an unpleasant $1 \mathrm{kHz}$ tone for a subgroup of six subjects. Activation of primary auditory cortices are overlaid on the normalization template. On the left, the fitted response and adjusted data are plotted as a function of peri-stimulus time for the most significant voxel in the left primary auditory cortex for two subjects. Activations are thresholded at $p<0.05$ (corrected).

We further investigated whether this temporally limited amygdala response is reflected in our behavioral SCR data. SCR for the first 8 and last 12 min of the conditioning phase were analyzed separately and contrasted. Figure 5D shows the differences of SCR for CS - and CS + unpaired for the early and late part of the conditioning phase, similar to Figure 2 . The significant interaction $(t[18]=2.5, p<0.05)$ indicates a relationship between lateral amygdala activation and behavioral measurements.

\section{Comparison of CS + and CS - Stimuli before Conditioning}

We assessed systematic effects related to the faces themselves by comparing responses elicited by the two conditioned and two neutral faces during the familiarization (preconditioning) phase, before any conditioning took place. This analysis confirmed that regions showing a significant activation for the $\mathrm{CS}+{ }_{\text {unpaired }}$ relative to the $\mathrm{CS}-$ in the conditioning phase showed no systematic differences between the faces at $p<0.001$ (uncorrected) in the familiarization phase of the experiment. We further tested the robustness of our principal findings by performing an interaction analysis, looking for differences between $\mathrm{CS}+{ }_{\text {unpaired }}$ and $\mathrm{CS}$ - in the familiarization relative to the conditioning phase. Although Z scores were in general smaller (as expected for interactions), this analysis confirmed the conditioning-specific effects reported above. We also tested for significant differences in amygdala adaptation (i.e., time by event interaction) between the conditioning phase and the familiarization phase, again looking explicitly for a difference between the familiarization and conditioning phases. This was again significant ( $p<0.001$, uncorrected). It should be emphasized that comparing activations during the conditioning relative to the familiarization phase (i.e., interaction) is only a supplementary analysis and could be confounded by temporal order effects. The more important comparison is between $\mathrm{CS}+_{\text {unpaired }}$ and $\mathrm{CS}-$ presented within the same trial. Furthermore, systematic effects of individual faces per se are unlikely, because we randomized the allocation of conditioned and neutral stimuli across subjects. 


\begin{tabular}{|c|c|c|c|c|c|}
\hline Coordinates & Z & $p$ value & Coordinates & Z & $p$ value \\
\hline Anterior cingulate left & & & right & & \\
\hline$-3,15,33$ & 6.3 & $<0.05 *$ & $6,30,42$ & 6.6 & $<0.05^{*}$ \\
\hline Red nucleus left & & & right & & \\
\hline$-6,-9,-6$ & 3.6 & $<0.001$ & $9,-21,-12$ & 4.6 & $=0.08 *$ \\
\hline \multicolumn{6}{|l|}{ Anterior insula left } \\
\hline$-33,27,-12$ & 8.0 & $<0.05 *$ & $51,24,-9$ & 8.0 & $<0.05^{*}$ \\
\hline \multirow[t]{2}{*}{ Medial parietal } & & & right & & \\
\hline & & & $15,-66,36$ & 5.6 & $<0.05^{*}$ \\
\hline Premotor left & & & right & & \\
\hline$-39,3,54$ & 4.6 & $=0.08^{*}$ & $48,12,39$ & 5.2 & $<0.05^{*}$ \\
\hline \multirow[t]{2}{*}{ SMA } & & & right & & \\
\hline & & & $6,6,54$ & 5.9 & $<0.05^{*}$ \\
\hline \multicolumn{3}{|c|}{$\begin{array}{l}\text { Time by condition interaction } \\
\text { Amygdala left }\end{array}$} & right & & \\
\hline$-24,3,-24$ & 4.6 & $<0.05^{* *}$ & $27,-3,-24$ & 3.2 & $<0.05^{* *}$ \\
\hline \multicolumn{6}{|c|}{$\begin{array}{l}\text { Differential activations comparing visual evoked responses for conditioned stimuli in the absence of an unconditioned stimulus (CS }+ \text { unpared } \\
\text { and CS }- \text {. Coordinates are in } \mathrm{mm} \text { according to Talairach and Tournoux (1988) and are based on spatial normalization to a template provided } \\
\text { by the Montreal Neurological Institute (Evans et al., 1994). } \\
* \text { p value corrected for entire brain volume. } \\
\text { ** v value corrected for volume of interest. }\end{array}$} \\
\hline
\end{tabular}

\section{Discussion}

In this study, we used event-related whole brain fMRI. This required a TR of $4.1 \mathrm{~s}$ and meant that we could only sample two to three time points per stimulus. We were, nevertheless, able to demonstrate sensory evoked responses in the visual and auditory system. Furthermore, we were able to demonstrate differential hemodynamic responses elicited by conditioned and neutral stimuli divorced from confounding effects of an associated aversive stimulus. These differences must reflect learning the association between a neutral stimulus and an aversive tone. The purely sensory characteristics of the stimuli themselves evoked identical responses in a familiarization phase. Activations revealed by comparing $\mathrm{CS}+{ }_{\text {unpaired }}$ against $\mathrm{CS}$ - within the same session are unlikely to reflect factors such as motivation. Although this might confound blocked designs, in the context of mixed trials as employed here motivation should affect all event types equally.

\section{Anterior Cingulate}

Bilateral, significant differentially evoked hemodynamic responses were found in the anterior cingulate cortex. This structure plays a crucial role in assessing the motivational content of internal and external stimuli and in regulating context-dependent behaviors (Devinsky et al., 1995) such as approach and avoidance learning (Freeman et al., 1996). Direct evidence for the participation of the anterior cingulate in classical conditioning comes from animal (Powell et al., 1996; Everitt and Robbins, 1997) and human (Schreurs et al., 1997) functional imaging studies. The latter study described positive correlations between changes in regional cerebral blood flow ( $\mathrm{rCBF}$ ) and percent conditioned responses. Cingulo-thalamic neuronal plasticity may be crucial for the acquisition of avoidance responses in the context of conditioning, and it has been suggested that amygdala projections play an important role in the modulation of these plastic changes (Poremba and Gabriel, 1997a, 1997b).

The affective role of the anterior cingulate has been dissociated from any perceptual stimulus-processing role (Rainville et al., 1997). This latter experiment demonstrated that $\mathrm{rCBF}$ in the anterior cingulate is linearly correlated to the affective intensity of mediated pain. A similar response behavior of the anterior cingulate is suggested by our results: the presentation of the unpaired CS + is not painful, but it has, through conditioning, become a predictor of pain (i.e., the expected ensuing unpleasant tone). In contrast to the study by Rainville et al. (1997), where hypnosis was used to downregulate pain affect, our experiment can be construed as effecting the reverse, namely, an upregulation of pain anticipation leading to a neural response in the absence of physical pain (for the CS+ $+_{\text {unpaired }}$ ).

This similarity of systems differentially activated in our experiment to those implicated in pain processing is remarkable. The activation of anterior insulae with anterior cingulate cortices is a prominent feature in most functional imaging studies of pain (Coghill et al., 1994; Rainville et al., 1997). It has been suggested that the anterior cingulate cortex together with the anteriorinsula provides one route through which nociceptive input is integrated with memory to allow appropriate responses to stimuli that predict future adversities (Coghill et al., 1994). This linkage of nociception with memory receives further support from our results. Activation of the red 


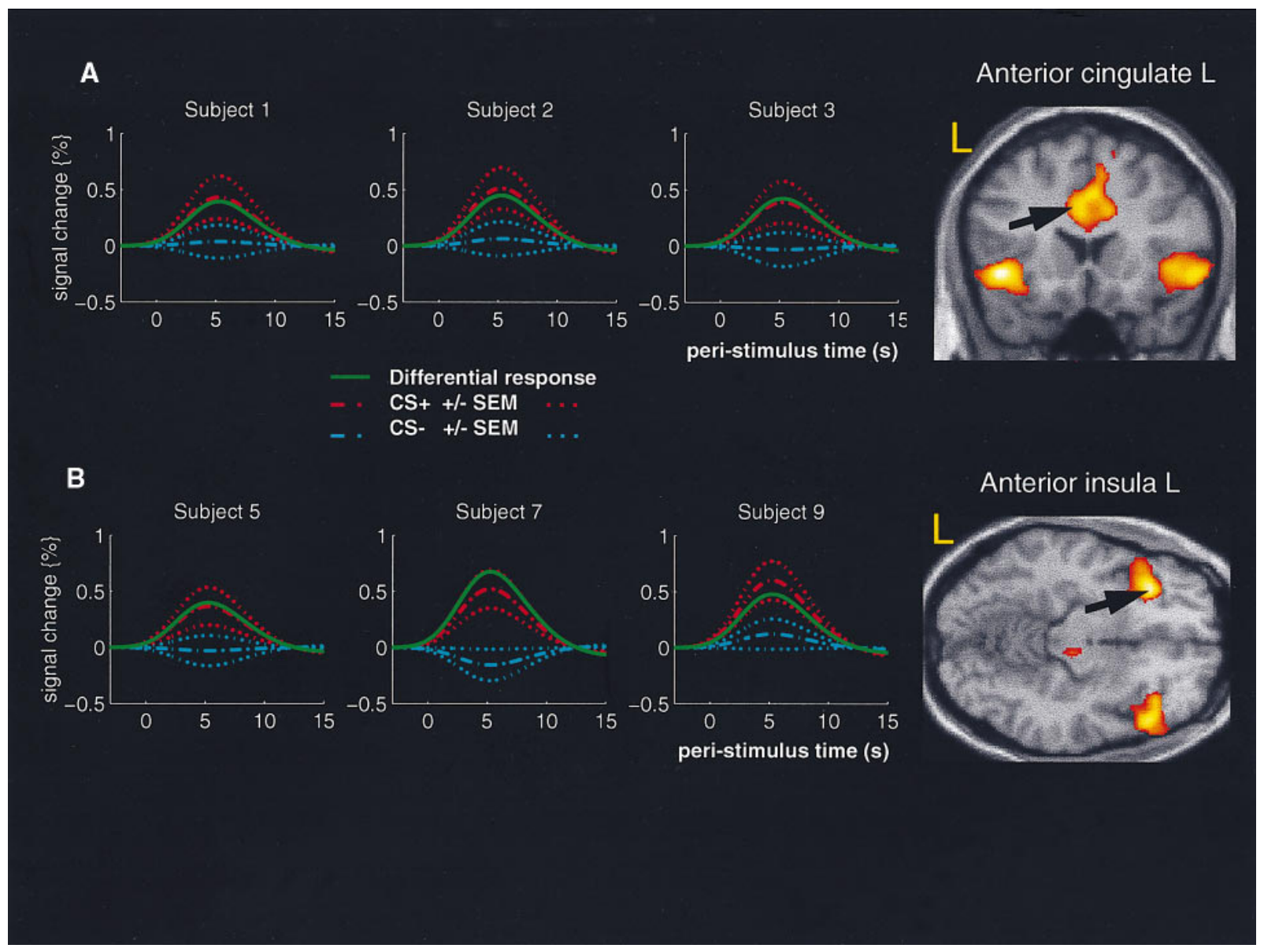

Figure 4. Neural Responses during Conditioning

(A) Significant differential visual evoked responses for conditioned versus neutral faces in the left anterior cingulate cortex $(x=-3, y=15$ $z=33 \mathrm{~mm}$ ). The coronal slice of the template T1 weighted MRI used for normalization shows differential responses in bilateral anterior insulae and right anterior cingulate. Images are thresholded at $p<0.001$ uncorrected for visualization. The significances for individual activations are given in Table 1. The peri-stimulus time plots are shown for three out of nine subjects. Instead of plotting raw data, we show the fitted response \pm the standard error of the mean (SEM) for CS - (blue) and CS $+_{\text {unpaired }}($ red) events. Dash-dotted lines represent the fitted response; dotted lines represent the mean \pm SEM. Statistical inference is based on the difference between the two responses, shown in green.

(B) Significant differential visual evoked responses for conditioned versus neutral faces in the left anterior insula $(x=-33, y=27, z=-12)$. The axial slice of a mean T1 weighted group MRI also shows differential responses in the right red nucleus and right anterior insula. Peristimulus time plots are shown for three out of nine subjects. Fitted responses \pm the standard error of the mean (SEM) for the CS - (blue) and $\mathrm{CS}+{ }_{\text {unpaired }}(\mathrm{red})$ events are shown. Statistical inference is based on the difference between the two responses, shown in green.

nuclei in this regard suggests that projections from the cingulate, which assimilate the value of a stimulus, interact with an efferent motor system as a means of regulating context-dependent behaviors.

\section{Anterior Insula}

Several studies suggest a role for the anterior insula in processing emotionally relevant contexts, for instance, disgust (Phillips et al., 1997), pain (Casey et al., 1995), and the recollection of affect-laden autobiographical information (Fink et al., 1996). This functional characterization is in keeping with known insular projections to anterior cingulate, perirhinal, entorhinal, and periamygdaloid cortices and various amygdaloid nuclei (Mesulam and Mufson, 1982). This pattern of connectivity, together with neurophysiological data, has led to a conceptualization of the insula as an area functionally associated with emotional processing (Casey et al., 1995; Augustine, 1996). In our study, the insula may provide information about primary reinforcers received from the sensory system (Rolls, 1994).

\section{Amygdala}

The initial analysis comparing CS + unpaired with CS - over the entire conditioning phase did not reveal amygdala activation. However, based on recent evidence from an electrophysiological study showing habituation of amygdala single cell responses during conditioning in the rat (Quirk et al., 1997), we performed a further analysis to explicitly test for habituation (time by event type interaction) of amygdala responses. In line with Quirk et al. (1997), the a priori hypothesis for this analysis was restricted to the amygdala. Correction for multiple comparisons in this analysis was based on the size of the 
A)

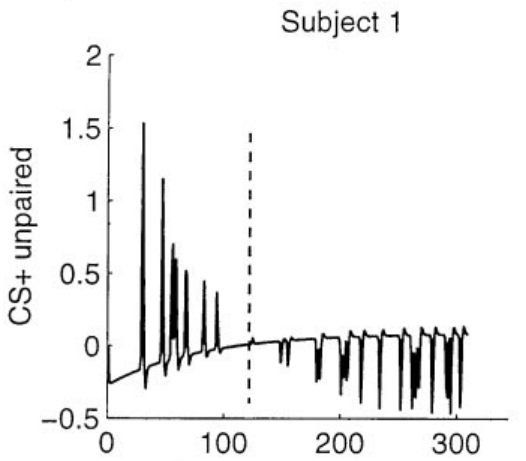

C)

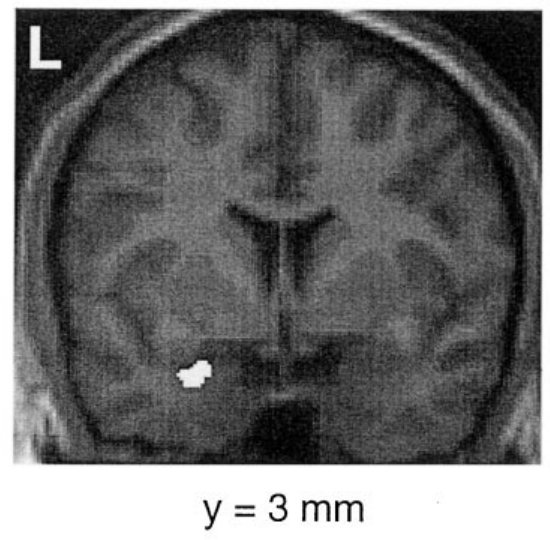

B)

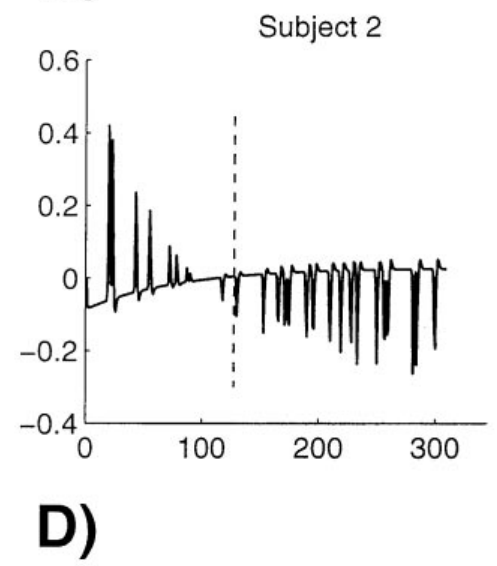

Interaction: SCR early versus late

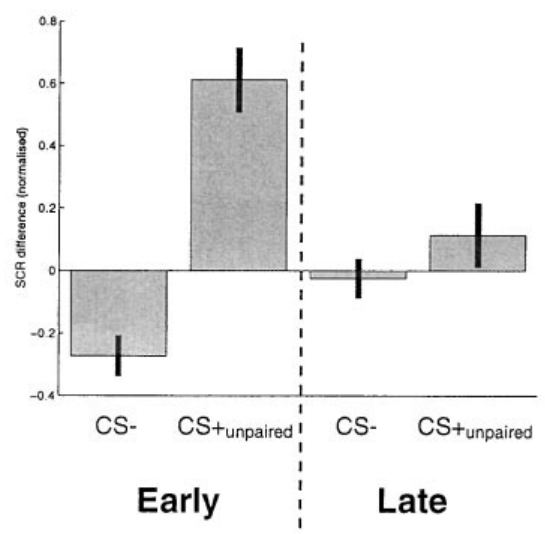

Figure 5. Time-Dependent Neural Responses in the Amygdala with Conditioning

Rapid habituation of amygdala responses to CS + unpaired stimuli. (A) and (B) show the fitted response for the CS + ${ }_{\text {unpaired }}$ events for two subjects

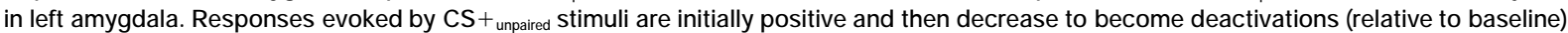
after 120 scans $(8.2 \mathrm{~min})$ for subject 1 and after 100 scans $(6.8 \mathrm{~min})$ for subject 2 . (C) shows the activation of the left amygdala ( $Z=4.6)$ overlaid on a template T1 MRI. Statistical inference is based on the difference between the time by condition interaction shown in (A) and (B) relative to responses evoked by CS - stimuli. (D) shows differences in SCR responses comparing the first 8 min to the last 12 min of the conditioning phase. The difference between SCR elicited by CS + unpaired versus CS - is clearly larger for the first 8 min of the experiment. This interaction is significant $(\mathrm{t}[18]=2.5, \mathrm{p}<0.05)$. The vertical dashed lines in $(A)$ and $(B)$ indicate the cutoff between early and late $(117$ volumes $=$ $8 \mathrm{~min}$ ) used for the SCR interaction analysis shown in (D).

amygdala as used by others (Breiter et al., 1996; Whalen et al., 1998). This anatomical constraint is justified on the grounds that ignoring this a priori evidence could lead to type II errors (Worsley et al., 1996).

In this additional analysis, the focus of maximal activation in the amygdala was seen in its rostrolateral part. Comparison of this location with response foci in other fMRI studies (Breiter et al., 1996; Whalen et al., 1998) reveals a difference in the $z$ coordinate values (ventraldorsal). Amygdala coordinates from these studies project into the striatum when using the template from the Montreal Neurological Institute as in our analysis. Therefore, we conclude that this difference is most likely due to differences in spatial normalization, for example, a different template. The activation of the lateral amygdala in classical conditioning is in accord with reciprocal projections between the medial geniculate nucleus and this part of the amygdala (LeDoux et al., 1990b). Furthermore, the lateral amygdala is strongly implicated in classical conditioning as shown by single cell recordings (Quirk et al., 1997) and lesion experiments (LeDoux et al., 1990a) in rats.

Amygdala responses to $\mathrm{CS}+{ }_{\text {unpaired }}$ stimuli showed rapid habituation. This accords with results from other neuroimaging studies showing rapid habituation of amygdala responses in the context of viewing emotionally expressive faces (Breiter et al., 1996; Whalen et al., 1998). Our results extend these observations to human classical conditioning and are in accord with animal data of amygdala responses to acoustic stimuli (Bordi and LeDoux, 1992; Bordi et al., 1993) and lateral amygdala responses in classical conditioning in the rat (Quirk et al., 1997). The transient response characteristic further suggests an important role for the amygdala during the 
early phase of aversive conditioning, consistent with the suggestion that the amygdala serves as a rapid subcortical information processing pathway for behaviorally relevant (e.g., dangerous) stimuli (LeDoux, 1996). This early versus late dissociation, initially suggested for the hippocampus (Alvarez and Squire, 1994), may also apply to the amygdala.

Lesion studies have stressed the importance of the amygdala in acquisition and expression of conditioned fear (Campeau and Davis, 1995). However, NMDA antagonist administration impairs acquisition and the associated emergence of plastic changes but not expression of conditioned responses (Miserendino et al., 1990), highlighting potential differences in underlying mechanisms. Recent studies of single neuron responses in the amygdala of awake, behaving rats also reveal striking temporal dynamics of amygdala responses (Quirk et al., 1997). Recordings from lateral amygdala show temporally limited neuronal responses during conditioning (Quirk et al., 1997), whereas cortical responses remain elevated throughout the conditioning phase. Amygdala responses in our experiment show similar temporally limited response characteristics.

However, the temporal pattern of amygdala responses in our study seems at odds with the lesion literature, where an intact amygdala is necessary not only for acquisition but also for expression of conditioned responses. One possibility is that plastic changes occurring during the early phase of conditioning are related to increased amygdala activity. In this context, the initial increases in amygdala activity might represent plastic changes, necessary for the acquisition of the conditioned response. Although the integrity of the amygdala is essential for the continued expression of conditioned responses, a refined connectivity within the amygdala and between the amygdala and other regions leads to lower mean firing rates and synaptic activity during later stages of conditioning.

Another possible explanation for the habituation of amygdala responses is based on the concept of negative feedback in classical conditioning (Fanselow, 1998). It has been demonstrated that fear conditioning using electric shocks as USs produces an amygdala-dependent analgesic state mediated by endogenous opioids (Fanselow, 1984). This can be seen as a negative feedback since analgesia leads to a reduced ability of the US to condition and explains habituation of amygdala responses in the study by Quirk et al. (1997). In our experiment, where an aversive tone serves as the US, engagement of the stapedius reflex might decrease sound transmission at the level of the middle ear and mediate negative feedback that reduces the impact of the US (Cacace et al., 1992). This conjecture accords with our behavioral data showing time-dependent differ-

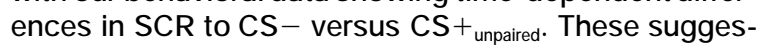
tions are necessarily speculative, and the functional significance of time-limited amygdala activations clearly warrants further investigations.

It has been speculated that the lateral amygdala serves as a differentiator and is therefore sensitive to changes in the CS-US relationship as it occurs at the beginning of conditioning (Quirk et al., 1997). On the other hand, ongoing selective activation of cortic al areas throughout conditioning is in accord with the proposal that cortical regions act as integrators. Our data showing habituation to the CS in the lateral amygdala and ongoing activation in cortical areas (e.g., anterior cingulate and insular) support this hypothesis and suggest a similar model for human associative learning. As already noted, the amygdala habituation is mirrored by the temporal characteristics of SCR, showing smaller differences between SCR to CS - versus CS + unpaired after 8 min of conditioning. This relation to behavioral measurements is in accord with a neuroimaging study showing correlations of amygdala activation during viewing emotionally arousing film clips with the number of recalled film clips after 3 weeks (Cahill et al., 1996).

The contribution of amygdala to conditioning may be linked to early modulation of cingulo-thalamic connectivity (Poremba and Gabriel, 1997a, 1997b). All of these observations suggest that with time, mnemonic representations of behaviorally salient contexts are expressed in cortical regions other than medial temporal lobe structures (McGaugh et al., 1996). Proposed candidate structures, including the anterior cingulate and insular cortices, were all activated in our study (Everitt and Robbins, 1997).

\section{Motor Structures: Red Nucleus, SMA, and Premotor Cortex}

Activation of the red nucleus together with premotor structures is typically associated with the efferent motor component of eyeblink conditioning (Clark and Lavond, 1993; Krupa et al., 1993; Thompson and Krupa, 1994; Logan and Grafton, 1995). The involvement of the red nucleus in this context has been established in animal studies of eyeblink conditioning (Desmond and Moore, 1991; Thompson and Krupa, 1994) and by functional imaging of humans (Logan and Grafton, 1995). Further evidence for an important role in conditioning comes from experiments in which reversible inactivation of the red nucleus blocked the expression of conditioned responses (Krupa et al., 1993). This is in contrast to cerebellar inactivation, which impairs acquisition of conditioned responses (Krupa et al., 1993; Ramnani and Yeo, 1996).

Subcortical motor nuclei and premotor activations raise the question of a relationship between classical conditioning, as employed in our experiment, and eyeblink conditioning. Lid closure in eyeblink conditioning is an obligatory defensive mechanism to avoid the impact of a US (e.g., air puff to the cornea). After conditioning, a previously neutral stimulus can elicit the same defensive response (i.e., blink). In our paradigm, subjects' ability to react was restricted by positioning in a tight head holder as well as by the instruction not to move during imaging. Although almost no actual movements occurred (confirmed by the estimated head motion of $<1.5 \mathrm{~mm}$ ), it is likely that generating a preparatory motor response is obligatory in this setting. In keeping with this suggestion, cortical activation patterns involving motor regions can be observed when movements are prepared or imagined but not executed (Decety et al., 1994; Stephan et al., 1995). 


\section{Conclusion}

Exploiting the high spatiotemporal resolution of $\mathrm{fMRI}$ for an event-related experimental design, our results illustrate the contribution of different cortical and subcortical areas to the rapid learning that mediates the behavioral effects of classical conditioning. Interpreting our results in the light of animal studies, we propose a central integrative role for the human "rostral limbic system," including anterior cingulate and insular cortices, which receive input from the amygdala, processing the behavioral relevance of sensory stimuli. Projections of the anterior cingulate to premotor regions and subcortical motor nuclei suggest a potential role for these structures in the formation of context-dependent adaptive behavioral responses.

\section{Experimental Procedures}

\section{Subjects and Imaging}

We studied nine healthy right-handed volunteers (seven male and two female). Written informed consent was obtained prior to MR scanning. Data were acquired with a 2 Tesla Magnetom VISION whole body MRI system (Siemens, Erlangen, Germany) equipped with a head volume coil. Contiguous multi-slice T2* weighted echoplanar images (TE $=40 \mathrm{~ms}, 80.7 \mathrm{~ms} /$ image, $64 \times 64$ pixels $[19.2 \mathrm{~cm} \times 19.2 \mathrm{~cm}]$ ) were obtained in an axial orientation. This sequence enhances blood oxygenation level-dependent (BOLD) contrast (Kwong et al., 1992). The volume acquired covered the whole brain (48 slices; slice thickness $3 \mathrm{~mm}$, giving a $14.4 \mathrm{~cm}$ vertical field of view). The effective repetition time (TR) was $4.1 \mathrm{~s} /$ volume.

During scans, subjects looked at a translucent screen through a $45^{\circ}$ angled mirror. The distance between eyes and screen was $\sim 300$ $\mathrm{mm}$. Visual stimuli (i.e., faces) were back-projected onto the screen by an LCD video-projector, subtending $17^{\circ}$ vertically and $8^{\circ}$ horizontally in the visual field. The screen refresh rate was set to 67/s. During the ITI, the screen was dark. Computer-generated auditory stimuli were delivered through plastic tubes, sealed by foam ear inserts. To further decrease the influence of the gradient switching noise from the scanner, the sound delivery system was shielded by plastic ear defenders. To minimize head motion, subjects were restrained with bitemporal pressure pads.

\section{Skin Conductance Responses}

We measured online SCRs in all subjects during $\mathrm{fMRI}$ scanning Unfortunately, SCR traces in four out of nine subjects could not be analyzed due to artifacts caused by gradient switching and radio frequency transmission from the MR scanner. SCRs were measured from two electrodes on the index and middle finger of the left hand using silver electrodes and electrode gel. The signal was amplified and sampled at $100 \mathrm{~Hz}$. Further offline processing was performed with MATLAB (The MathWorks, Natick, MA). Data were detrended and temporally smoothed (Gaussian kernel with full width at half maximum of $2 \mathrm{~s}$ ), and remaining MRI scanning artifacts were eliminated. Finally, the time series were resampled at $10 \mathrm{~Hz}$ using sinc interpolation. For quantitative analysis of SCRs (similar to Esteves et al., 1994), evoked SCR responses were characterized by the maximum of the SCR signal in the interval between stimulus onset and $5 \mathrm{~s}$ after stimulus onset. Extending this window beyond the onset of the US ( $3 \mathrm{~s}$ ) was possible since we only analyzed CS - and $\mathrm{CS}+$ unpaired. This value was then subtracted from a baseline, the mean of the SCR in the second before the onset of the stimulus, to account for residual baseline fluctuations. For statistical analysis, the differences were normalized to zero mean and standard deviation of unity. The significance of $\mathrm{SCR}$ differences for $\mathrm{CS}-$ and $\mathrm{CS}+{ }_{\text {unpaired }}$ was assessed by a t test. We analyzed both groups (SCRs acquired during fMRI scanning and SCRs acquired in a separate session) separately to demonstrate that conditioning took place during scanning. The group of subjects that was tested after fMRI scanning comprised three of the fMRI study participants. The fourth imaging volunteer being unavailable, we substituted him with another subject.

\section{Image Processing and Statistical Analysis}

Image processing and statistical analysis were carried out using SPM 97 (Worsley and Friston, 1995; Friston et al., 1995b; see also http://www.fil.ion.ucl.ac.uk/spm). The problem of medial temporal lobe susceptibility artifacts was addressed by looking at animated sequences of raw images. This procedure revealed no event-related changes in susceptibility. All volumes were realigned to the first volume (Friston et al., 1995a). Residual motion effects were eliminated at each and every voxel by regressing a periodic function of the estimated movement parameters at each voxel on the time course of this voxel. Actual head movements were $<1.5 \mathrm{~mm}$ for all subjects. To account for the different sampling times of different slices, voxel time series were interpolated using sinc interpolation and resampled using the slice at the $\mathrm{AC}-\mathrm{PC}$ line as the reference. A mean image was created using the realigned volumes. An anatomical MRI, acquired using a standard three-dimensional T1 weighted sequence $(1 \times 1 \times 1.5 \mathrm{~mm}$ voxel size), was coregistered to this mean (T2*) image. This measure ensured that the functional and structural images were spatially aligned. Finally, the structural image was spatially normalized (Friston et al., 1995a) to a standard template (Talairach and Tournoux, 1988; Evans et al., 1994), using nonlinear basis functions. The nonlinear transformation mapping the structural T1 MRI scan onto the template was also applied to the fMRI data. The data were smoothed using an $8 \mathrm{~mm}$ (full width at half maximum) isotropic Gaussian kernel to compensate for residual variability after spatial normalization and to permit application of Gaussian random field theory to provide corrected statistical inference (Friston et al., 1995c).

\section{Conditioning}

The data were analyzed by modeling the evoked hemodynamic responses for different stimuli as delta functions convolved with a synthetic hemodynamic response function in the context of the general linear model as employed by SPM 97 (J osephs et al., 1997). We defined three different event types: all were time-locked to the onset of the presentation of a face. The events were subdivided into (1) CS - (2) CS + paired (CS + paired), and (3) CS + unpaired $(C S+$ unaired) face stimuli (Figure 1). Specific effects were tested by applying appropriate linear contrasts to the parameter estimates for each event, resulting in a t statistic for every voxel. These $t$ statistics (transformed to Z statistics) constitute an SPM. The contrast used in the main analysis tested for greater responses evoked by $\mathrm{CS}+$ unpaired stimuli relative to $\mathrm{CS}$-.

\section{Sensory (Auditory and Visual) Evoked Responses}

In this analysis, we added a fourth event type to the main analysis, which was time-locked to the presentation of the US ( $1 \mathrm{kHz}$ tone). The contrasts for the auditory evoked responses tested for the comparison of these events to baseline. For the visual evoked responses, the contrast resembles the grouped comparison between CS -, CS $+_{\text {unpaired, }}$ and CS $+_{\text {paired }}$ to baseline. For this analysis, only the first six subjects were considered.

\section{Interaction Analysis Including Familiarization Trials}

We also combined the data for the familiarization phase and the conditioning phase and defined two additional events for the familiarization phase (fCS - and $\mathrm{fCS}+$ ) for the faces that will become $\mathrm{CS}-$ and $\mathrm{CS}+$. The contrast in this analysis modeled the difference between $\mathrm{fCS}+$ versus $\mathrm{fCS}-$ and $\mathrm{CS}+{ }_{\text {unnared }}$ versus $\mathrm{CS}-$, that is, the interaction.

\section{Time by Event Interaction Analysis}

In addition to the main analysis, we defined three new regressors representing the time by event interactions. These three additional regressors were created by multiplying the regressors for CS-, $\mathrm{CS}+$ paired, and CS + unpaired with a mean corrected exponential function with a time constant of one-fourth of the session length. Contrasts tested for the difference of the interaction terms between the CSand $\mathrm{CS}+$ unpaired.

Ensuing SPMS were interpreted by referring to the probabilistic behavior of Gaussian random fields. The data for all nine subjects were analyzed as a group, modeling evoked responses in a subjectspecific way. To demonstrate individual effects, we display the evoked hemodynamic response for contrasts of interest for subjects 
individually. In the case of the amygdala, where we had a regionspecific hypothesis, correction for multiple comparisons was based on the volume of interest (Filipek et al., 1994) and the smoothness of the underlying SPM (Worsley et al., 1996). For the remaining brain, where we had no a priori regional hypothesis, correction was for the entire volume analyzed (i.e., whole brain). Thus, in all cases the threshold was set to $p<0.05$.

\section{Acknowledgments}

We thank A. Kleinschmidt, N. Ramnani, D. McGonigle, and R. Frackowiak for helpful comments. We would also like to thank the Functional Imaging Laboratory physics group and the radiographers for their support and help. C. B., J.M., R. D., and K. J . F. are funded by the Wellcome Trust.

Received February 4, 1998; revised April 29, 1998.

\section{References}

Alvarez, P., and Squire, L.R. (1994). Memory consolidation and the medial temporal lobe: a simple network model. Proc. Natl. Acad. Sci. USA 91, 7041-7045.

Augustine, J.R. (1996). Circuitry and functional-aspects of the insular lobe in primates including humans. Brain Res. Rev. 22, 229-244.

Bechara, A., Tranel, D., Damasio, H., Adolphs, R., Rockland, C., and Damasio, A.R. (1995). Double dissociation of conditioning and declarative knowledge relative to the amygdala and hippocampus in humans. Science 269, 1115-1118.

Bordi, F., and LeDoux, J . (1992). Sensory tuning beyond the sensory system-an initial analysis of auditory response properties of neurons in the lateral amygdaloid nucleus and overlying areas of the striatum. J. Neurosci. 12, 2493-2503.

Bordi, F., LeDoux, J ., Clugnet, M.C., and Pavlides, C. (1993). Singleunit activity in the lateral nucleus of the amygdala and overlying areas of the striatum in freely behaving rats-rates, discharge patterns, and responses to acoustic stimuli. Behav. Neurosci. 107, 757-769.

Breiter, H.C., Etcoff, N.L., Whalen, P.J ., Kennedy, W.A., Rauch, S.L., Buckner, R.L., Strauss, M.M., Hyman, S.E., and Rosen, B.R. (1996). Response and habituation of the human amygdala during visual processing of facial expression. Neuron 17, 875-887.

Buckner, R.L., Bandettini, P.A., Ocraven, K.M., Savoy, R.L., Petersen, S.E., Raichle, M.E., and Rosen, B.R. (1996). Detection of cortical activation during averaged single trials of a cognitive task using functional magnetic-resonance-imaging. Proc. Natl. Acad. Sci. USA 93, 14878-14883.

Cacace, A.T., Margolis, R.H., and Relkin, E.M. (1992). Short-term poststimulatory response characteristics of the human acoustic stapedius reflex-monotic and dichotic stimulation. J. Acoust. Soc. Am. 91, 203-214.

Cahill, L., Haier, R.J ., Fallon, J ., Alkire, M.T., Tang, C., Keator, D., Wu, J., and McGaugh, J.L. (1996). Amygdala activity at encoding correlated with long-term, free-recall of emotional information. Proc. Natl. Acad. Sci. USA 93, 8016-8021.

Campeau, S., and Davis, M. (1995). Involvement of the central nucleus and basolateral complex of the amygdala in fear conditioning measured with fear-potentiated startle in rats trained concurrently with auditory and visual conditioned-stimuli. J . Neurosci. 15, 23012311.

Casey, K.L., Minoshima, S., Morrow, T.J ., Koeppe, R.A., and Frey, K.A. (1995). Imaging the brain in pain-potentials, limitations, and implications. Adv. Pain Res. Ther. 22, 201-211.

Clark, R.E., and Lavond, D.G. (1993). Reversible lesions of the red nucleus during acquisition and retention of a classically-conditioned behavior in rabbits. Behav. Neurosci. 107, 264-270.

Coghill, R.C., Talbot, J .D., Evans, A.C., Meyer, E., Gjedde, A., Bushnell, M.C., and Duncan, G.H. (1994). Distributed-processing of pain and vibration by the human brain. J. Neurosci. 14, 4095-4108.
Dale, A.M., and Buckner, R.L. (1997). Selective averaging of rapidly presented individual trials using fMRI. Hum. Brain Map. 5, 329-340. Decety, J., Perani, D., J eannerod, M., Bettinardi, V., Tadary, B., Woods, R., Mazziotta, J.C., and Fazio, F. (1994). Mapping motor representations with positron emission tomography. Nature 371, 600-602.

Desmond, J.E., and Moore, J.W. (1991). Single-unit activity in red nucleus during the classically-conditioned rabbit nictitating-membrane response. Neurosci. Res. 10, 260-279.

Devinsky, O., Morrell, M.J., and Vogt, B.A. (1995). Contributions of anterior cingulate cortex to behavior. Brain 118, 279-306.

Ekman, P. (1982). Emotion in the Human Face (Cambridge: Cambridge University Press).

Esteves, F., Parra, C., Dimberg, U., and Ohman, A. (1994). Nonconscious associative learning - pavlovian conditioning of skin-conductance responses to masked fear-relevant facial stimuli. Psychophysiology 31, 375-385.

Evans, A.C., Kamber, M., Collins, D.L., and Macdonald, D. (1994), An MRI-based probabilistic atlas of neuroanatomy. In Magnetic Resonance Scanning and Epilepsy, S. Shorvon, D. Fish, F. Andermann, G. M. Bydder, H. Stefan, eds. (New York: Plenum Press), pp. 263-274.

Everitt, B.J ., and Robbins, T.W. (1997). Central cholinergic systems and cognition. Annu. Rev. Psych. 48, 649-684.

Fanselow, M.S. (1984). What is conditioned fear. Trends Neurosci. 7, 460-462.

Fanselow, M.S. (1998). Pavlovian conditioning, negative feedback, and blocking: mechanisms that regulate association formation. Neuron 20, 625-627.

Filipek, P.A., Richelme, C., Kennedy, D.N., and Caviness, V.S. (1994). Young-adult human brain-an MRI-based morphometric analysis. Cerebral Cortex 4, 344-360.

Fink, G.R., Markowitsch, H.J ., Reinkemeier, M., Bruckbauer, T., Kessler, J., and Heiss, W.D. (1996). Cerebral representation of ones own past-neural networks involved in autobiographical memory. J. Neurosci. 16, 4275-4282.

Fredrikson, M., Wik, G., Fischer, H., and Andersson, J . (1995). Affective and attentive neural networks in humans-a pet study of pavlovian conditioning. Neuroreport 7, 97-101.

Freeman, J .H., Cuppernell, C., Flannery, K., and Gabriel, M. (1996). Limbic thalamic, cingulate cortical and hippocampal neuronal correlates of discriminative approach learning in rabbits. Behav. Brain Res. 80, 123-136.

Friston, K.J ., Ashburner, J ., Frith, C.D., Poline, J .-B., Heather, J .D., and Frackowiak, R.S.J . (1995a). Spatial registration and normalization of images. Hum. Brain Map. 2, 1-25.

Friston, K.J ., Holmes, A.P., Poline, J.-B., Grasby, P.J., Williams, S. C. R., Frackowiak, R.S.J ., and Turner, R. (1995b). Analysis of fM RI time series revisited. Neurolmage 2, 45-53.

Friston, K.J ., Holmes, A.P., Worsley, K.P., Poline, J .-B., Frith, C.D., and Frackowiak, R.S.J . (1995c). Statistical parametric maps in functional imaging: a general linear approach. Hum. Brain Map. 2, 189-210.

J osephs, O., Turner, R., and Friston, K. (1997). Event-related fMRI. Hum. Brain Map. 5, 243-248.

Krupa, D.J ., Thompson, J .K., and Thompson, R.F. (1993). Localization of a memory trace in the mammalian brain. Science 260, 989-991.

Kwong, K.K., Belliveau, J.W., Chesler, D.A., Goldberg, I.E., Weisskoff, R.M., Poncelet, B.P., Kennedy, D.N., Hoppel, B.E., Cohen, M.S. Turner, R., et al. (1992). Dynamic magnetic resonance imaging of human brain activity during primary sensory stimulation. Proc. Natl. Acad. Sci. USA 89, 5675-5679.

LaBar, K.S., and LeDoux, J .E. (1996). Partial disruption of fear conditioning in rats with unilateral amygdala damage-correspondence with unilateral temporal lobectomy in humans. Behav. Neurosci. $110,991-997$.

LeDoux, J. (1996). A few degrees of separation. In The Emotional Brain. (New York: Simon and Schuster), pp. 138-178. 
LeDoux, J.E., Cicchetti, P., Xagoraris, A., and Romanski, L.M. (1990a). The lateral amygdaloid nucleus: sensory interface of the amygdala in fear conditioning. J. Neurosci. 10, 1062-1069.

LeDoux, J .E., Farb, C.F., and Ruggiero, D.A. (1990b). Topographic organization of neurons in the acoustic thalamus that project to the amygdala. J. Neurosci. 10, 1043-1054.

Logan, C.G., and Grafton, S.T. (1995). Functional-anatomy of human eyeblink conditioning determined with regional cerebral glucosemetabolism and positron-emission tomography. Proc. Natl. Acad. Sci. USA 92, 7500-7504.

McGaugh, J .L., Cahill, L., and Roozendaal, B. (1996). Involvement of the amygdala in memory storage-interaction with other brain systems. Proc. Natl. Acad. Sci. USA 93, 13508-13514.

Mesulam, M.M., and Mufson, E.J. (1982). Insula of the old-world monkey. 3. Efferent cortical output and comments on function. J. Comp. Neurol. 212, 38-52.

Miserendino, M.J.D., Sananes, C.B., Melia, K.R., and Davis, M. (1990). Blocking of acquisition but not expression of conditioned fear-potentiated startle by NMDA antagonists in the amygdala. $\mathrm{Na}$ ture $345,716-718$

Morris, J .S., Friston, K.J ., and Dolan, R.J . (1997). Neural responses to salient visual stimuli. Proc. R. Soc. Lond. B. 264, 769-775.

Phillips, M.L., Young, A.W., Senior, C., Brammer, M., Andrew, C., Calder, A.J ., Bullmore, E.T., Perrett, D.I., Rowland, D., Williams, S.C.R., et al. (1997). A specific neural substrate for perceiving facial expressions of disgust. Nature 389, 495-498.

Poremba, A., and Gabriel, M. (1997a). Amygdalar lesions block discriminative avoidance learning and cingulothalamic training-induced neuronal plasticity in rabbits. J . Neurosci. 17, 5237-5244.

Poremba, A., and Gabriel, M. (1997b). Medial geniculate lesions block amygdalar and cingulothalamic learning-related neuronal activity. J . Neurosci. 17, 8645-8655.

Powell, D.A., Maxwell, B., and Penney, J . (1996). Neuronal-activity in the medial prefrontal cortex during pavlovian eyeblink and nictitating-membrane conditioning. J . Neurosci. 16, 6296-6306.

Quirk, G.J ., Armony, J .L., and LeDoux, J .E. (1997). Fear conditioning enhances different temporal components of tone-evoked spike trains in auditory cortex and lateral amygdala. Neuron 19,613-624.

Rainville, P., Duncan, G.H., Price, D.D., Carrier, B., and Bushnell, M.C. (1997). Pain affect encoded in human anterior cingulate but not somatosensory cortex. Science $277,968-971$.

Ramnani, N., and Yeo, C.H. (1996). Reversible inactivations of the cerebellum prevent the extinction of conditioned nictitating-membrane responses in rabbits. J. Physiol. 495, 159-168.

Rolls, E.T. (1994). A theory of emotion and consciousness, and its application to understanding the neural basis of emotion. In The Cognitive Neurosciences, M.S. Gazzaniga, ed. (Cambridge, MA: MIT Press), pp. 1091-1106.

Rolls, E.T., Hornak, J ., Wade, D., and McGrath, J . (1994). Emotionrelated learning in patients with social and emotional changes associated with frontal-lobe damage. J. Neurol. Neurosurg. Psychiatry 57, 1518-1524.

Schreurs, B.G., McIntosh, A.R., Bahro, M., Herscovitch, P., Sunderland, T., and Molchan, S.E. (1997). Lateralization and behaviora correlation of changes in regional cerebral blood flow with classical conditioning of the human eyeblink response. J . Neurophysiol. 77, 2153-2163.

Stephan, K.M., Fink, G.R., Passingham, R.E., Silbersweig, D., Ceballosbaumann, A.O., Frith, C.D., and Frackowiak, R.S.J . (1995). Functional-anatomy of the mental representation of upper extremity movements in healthy-subjects. J . Neurophysiol. 73, 373-386.

Talairach, P., and Tournoux, J . (1988) A Stereotactic Coplanar Atlas of the Human Brain (Stuttgart: Thieme).

Thompson, R.F., and Krupa, D.J . (1994). Organization of memory traces in the mammalian brain. Annu. Rev. Neurosci. 17, 519-549.

Whalen, P.J ., Rauch, S.L., Etcoff, N.L., McInerney, S.C., Lee, M.B. and J enike, M.A. (1998). Masked presentations of emotional facial expressions modulate amygdala activity without explicit knowledge. J . Neurosci. 18, 411-418.
Worsley, K.J ., and Friston, K.J . (1995). Analysis of fMRI time series revisited-again. Neurolmage 2, 173-181.

Worsley, K.J ., Marrett, P., Neelin, A.C., Friston, K.J ., and Evans, A.C. (1996). A unified statistical approach for determining significant signals in images of cerebral activation. Hum. Brain Mapp. 4, 58-73. 